\title{
PARALISIS ASCENDENTE AGUDA
}

\author{
Por los Dres. MARIO GONZALEZ R. y CARLOS ALLENDE \\ Hospital Roberto del Rio. Cátedra Prof. A. Scroggie.
}

La parálisis ascendente aguda lleva incluída en si un grupo de enfermedades o sindromes. Asi es como en ella podrían englobarse: la parálisis de Landry, la poliomielitis ascendente, el sindrome de Guillain-Barré y las polineuritis de evolución sobreagtida. El objeto de este trabajo es pretsentar 4 casos de parálisis ascenjente aguda. 2 de Landry, 1 Guillain-Barré y $l$ de poliomielitis ascendente aguda; hemos elegido un solo caso de Heine-Medin, no porque éste haya sido el único que hemos observado, sino por ser demasiado conocido de todos los médicos, ya que según W:ckman, llega a constituir e1 $6 \%$ de las poliomielitis.

Otro de los fines de nuestras colaboración a este apasionante tema, es establecer la diferencia que ex ste entrel una parálisis de Landry y la parálisis infantil ascendentel ya que son muchos los autotes que las incluyen a ambas en la misma terminología.

\section{HISTORIA}

Heine de Cannstadt, al descr.bir en 1840 la poliomielitis como enfermedad autónoma, consideró a la parálisis ascendente aguda como una de sus formas clínicas. Landry J. B. en 1859 publica una interesante observación sobre esa enfermedad, é insiste este autor en algunos caracteres c'ínicos tales como, evolución fatal por parálisis de los músculos respiratorios, negatividad del líquido céfalo raquídeo. Posteriormente se han agregalo otros trabajos sobres esta materia. Landsteiner y Levaitti, en 1910. consiguieton producir pa rálisis ascendente aguda inćculando a monos. viras de po. Jiomielitis. 
Guillain-Barré, en 1916, aislan de étos cuadros clinicos un sindrome con caraiteres bien definidos, entre los que hacen resaltar, su evolución favorable y la discciación albúminocitológica en ell liquido céfalo raquídeo.

Cuadros clinicos semejantes al descrito por Landry han observado otros autores.

Rolleston to encuentra como complicación en algunas difterias; recalca él la presencia concomitante de parálisis del velo del palladar.

Remlinger describe la parálisis ascendente aguda como complicación en la vacunación antirráb:ca. Knutti hace notar la presencia de este mismo tipo de parálisis en la rabia.

Box, C. R., pone en evidencia este tipo de parálisis en el curso del sarampión. Mason la encuentra en el curso de la hematoporfirinuria agudà idiopática. Ultimamente Thorner, Alpers y Yaskía publican un trabajo sobre patálisis de Landry insistiendo bien en las características que anotaremos posteriormente.

\section{DEFINICION}

La parálisis de Landry es un sindrome y como tal es difícil establecer una def nición precisa. Este término se ha empleado con demasiada liberalidad y muchos autores han sido pródigos en ciasificar como tal. a toda parálisis que sé instala en forma brusca y es rápidamente ascendente. sin tomar en cuenta una base anatómica; otros la interptetan como un tipo de polineuritis sobrol-aguda y no la reconocen ni siquiera como entidad distinta.

Sin embargo, si nos circunscribimos estristamente a los casos descritos por Landry, podriamos definirla, siguiendo a Ford. en la siguiente forma: "Es un sindrome que sel caracteriza por la aparición brusca de parálicis flácida, que se desarrolla rápidamente. de evolución ascentionte y que compromete los distintós grupos musculares en el mismo orden en que están representados en la medula espinal. Notable es en él la falta de trastornos de la sensibilidad y del psiquis, así como tampoco hay temperatura alta; un hecho más notorio aún. es la presencia del liquic'o céfalo raquídeo normal o con muy leves alterariones inflamatorias. Los hallazgos anatómicos son también nutos $y$ por regla general, lo único que se encuentra. es cromatolicis de las células motoras y esto en los casos que han evolucionado en más de tres días". 
Considerándoto en forma global en un $30 \%$ dei los casos no encontramos una base anatómica (Thorner y col.).

Posteriormente se han agregado a este cuadro clínico, sindromes de ettiología bien definióa, pero con caracteristicas semejantes.

\section{ETIOLOGIA}

Para simplificar haremos una clas:ficación de los factotes más importantes de la parálisis de Landry:

a) Infecciones. Se le ha descrito en el curso de algunas enfermedades como la tifoídea, difteria. neumonía, septicemias, influenza, tifus exantemático de las Rocalloras (tic fever), rabia, sarampión.

b) Vacunaciones. Se le ha evidenciado en el curso de la vacunación antirrábica, interpretándosel su acción como reacción del tejido nervioso a otro tejido semejante heterólogo. más que por acción del virus rábico, su pronóstico es bueno, curando alreidedor d2: $80 \%$ totalmente. También se le ha descrito en la vacunación antitetánica.

c) Intoxicaciones. Princ palmente en el alcoholismo agudo y en la producida por plomo.

-d) Traumatismos.

e) Sin causa conocida.

\section{ANATOMIA PATOLOGICA}

Es sumamente variable. Lo frecuentel es encontrat sólo congestión muy discreta. En un número pequeño de casos hay lesiones de tos nervios perifér cos. En alrededor del $36 \%$ de los casos hay alteraciones de la medula espinal. En un $15 \%$ las lesiones són combinadas en ambas partes. Cuando es la medu'ia la afectada, son las células del cuerno anterior las que sufren ptincipalmente. El tipo de las lesiones puede ser inflamatorio o degenerativo. Las células puéden presentarse hinchadas, con los núcleos desplazados, las substancia de Nissl deshecha, en parte como polvitlo, desplazada a la periferia, déjando una zona clara alrededor del núcleo.

Sin embargo do constante es no encontrar lesiones anátomo-patológicas; éstas se encuentran en pacientes que sobreviveln un tiempo mayor de cinco dias.

En tesumen podemos- decir que en el estudio anátomohisto-patológico de la parálisis de Landry, no hay nada que sugiera una alteración específica. 


\section{CUADRO CLINICO}

Es una enfermeciad rara. que se presenta con más frecuencia por encima de los quince años.

Entre los sintomas prodrómicos se han descrito el decaimiento, la anorexia $y$ cierta laxitud de los miembros. I uego comienzan las parálisis en forma brusca. iniciándose en los miembros inferiores. El enfermo acusa primero sensación de rigidez o de pereza en las piernas (lo que constituye una falsa interptetación de la hipotonía). La parálisis es flácida y simétrica. Progresa rápidamente hacia arriba. siguiendo el orjen correspondiente a su representación en la médula espinal. Si la parálisis no es completa. aparecen como más comprometidos ios músculos proximales que los distales y esto se explica lógicamente, pust estos no tienen que mover toda la extremidad. Junto a lo deccrito tenemos marcada hipotonía e hiporreilexia y en muy pocos cases hay tras. tornos esfinterianos (mielitis agtegada). No hay dolor. El psiquis está normal o muy ligeramente alterado, 'a tempera. tura es normat o apenas subfebri?. Si practicamos algunos exámenes como orina, hemograma. atos son normales.

El indivi no muere por parálisis respirator a o por un proccso infeccicso agregado (bronconeumonia).

Ya lo dijimos anteriormente que en el examen del liquido céfalo-raquides lo conctante era no encontrar modif:caciones.

En caso de detenerse la parálisis antes qual se tomen 'os contror vitales. el enfermo sobrevive, prcduciéndose atrofjas musculares $\mathrm{y}$ cambios in las reaccioner electritas qie antes no se precentarón. Al sticeder esto el paciente mejora relativamente rápido. en 3 a 4 meses, mucho más que lo; que sucederia $a_{i}^{\prime}$ tratarse de una po:ineuritis de evolución sobreaguda.

\section{DIAGNOSTICO}

El fundamento del diagnóstico 'o tenemos an la defini ción qua dimos de la parális's de Landry. Es preciso diferenciarla ce algunas enfermedades como:

1) Poliomielitic, quo en su fase aguda pted: dar una sintomatologia semejante. pero dn el Heine-Medin tenemos síntomas prodrómico: más o menos característicos como los dolores musculates. la sudoración, posteriormente hay fiebre. alteraciones vésico-rectales, modificaciones en el líquido céfalo raquídeo (reacciones de las g.óculinas positivas intensas, leu- 
cocitosis con linfocitosis); rara vez hay compromiso de todos los músculos.

2) Encéfalomielitis a virus filtrable, que presenta una sintomatología bieln cefinida (hipertonía signos clínicos encefálicos, etc.).

3) Mielitis transversa aguda, en la que encontramos anestesia bajo la lesión. Babinsky positivo, pérdida del control de los esfinteres.

4) Con la polineuritis sobre-aguda es sumamente dificil establecer un diagnóstico diferencial. Antériormente habíamos dicho que algunos autores consideran la parálisis de Landry como una simple polineuritis, sin elmbargo esta enfermedad, si bien es cierto que comienza en las articulacicnes distales, al progresar, lo bace en forma anárquica. sin tomat en cuenta su representación en el segmento medular, además es rato que se tomen los músculos del tronco.

5) Sindrome de Guillain-Barré, dado a conocer por primera vez por estos autores en 1916; publicaron dos casos en adultos, con los siguientels caracteres: Es una politradículoneuritis de evolución favorable, en la que faltan graves alteraciones tróficas, acompañada de disociación de los reflejos (abolición del los tendinosos, con conservación de los cutáneos), y un hecho que es característico. como es la disociación albúnimo-citológica, semejante a! sindrome de Sicard, en el liquido espinal.

6) Polineuritis apiólica o polinkuritis de Teer Braak. producida por el fosfato de tri-orto-cresol, que se encuentra en ä̈gunas bebidas gaseosas, como el ginger-ale y también en la crelosota, produce parálisis que afectan principalmente las partes distales, sin alteraciones sensitivas.

7) Parátisis periódica familiar o parálisis de Wesphal. que aparece durante la noche, no compromete los etsínteres, la sensibilidad está normal y dura sólo algurias horas para reaparecer después de aigún tempo.

8.) Meningo-miclitis lútica de etiología conocida y con sintomas muy diferentes.

\section{PRONOSTICO}

En general ec una enfermedad grave, mueren alrededor del $80 \%$ del los enfermos, ya seà por compromiso de los músculos respiratorios, de los centros vitales o por una bronconeumonia agregaća. 
Un hecho curioso de anotar es que en los casos raros en que evoluciona a la curación. no deja secuelas y la reintegración es total dentro de 3 a 6 meses. Se han descrito recaidas y recidivas.

\section{TRATAMIENTO}

Actualmente no tiene un tratamiento específico. Apoyándose algunos autotẹs en la hipótesis de que sea ana polineuritis de evolución sobreaguda, han indicado la vitamina $\mathrm{B}^{1}$, en altas dosis $y$ principalmante por vía raquidea. sin embargo los éxitos que se describan son discutibles, por tratarse de una enfermedad que en ocasiones regresa espontáneamente, aun en casos que parecían desesperados.

Como medidas generales se recomienda el aislamiento, que es sumamente benéfico, pues además de actuar sobre el psiquis del enfermo, lavorece la aplicación géel la terapéutica que más adelante describiremos. En segundo lugar es necesario evitar ell enfrianiento, emp'eando todos los medios conocidos para este objeto.

Es la acumulación de secreciones en las vias respiratorias uno de los factores que agravan el pronóstico de esta afec. ción. ya sea por provocar una asfixia por obstrucción a nivel de la tráquea o bronquios o bien una bronconelumonía por aspiración; de ahí que sea recesario eliminarlas. Para ello se aconseja colocar al paciente en posición cét Trendelenburg. medida que muchas veces es insuficiente; entonces debemos rekurrir a un aspirador apropiado y al no contar con él, debemos extraerla con tina jeringa a la que se añade una sonda. método bastante primitivo, pero que én un caso urgente puede prektar su ayuda.

Si las secreciones son muy abundantes y sobre todo si scjn muy viscosas (por la polipnea), y existe el eminente peligro de asfixia se hace indispinsable recurrir a la traqueotomía, oue favorece su expulsión o su aspiración.

La oxígenoterapía y la respiración artificial desempeñan un importante papel en la t:trapéutica de esta grave enfermedad; esta última se puede practicar por medio del pulmotor o pulmón de acero de Drinker. con eil cual es posible mantener durante dias a un enfermo con sus músculos respiratorios paralizados.

Durante la evolución de! periošo paralítico. debelmos evitar las actitudes y posiciones viciosas. También se aconseja la vitamina $E$ que actuaría en el trofismo muscular. 
Ante un enfermo de parálisis de Landry debemos recurrir a todas las medidas enunciadas, sin perder jamás la esperanzà, sin abandonar un momento al que la sufre, pues si rápida es en aparecelr y en progresar, así tamb:én rápidamente sue'e regresar.

\section{CASOS CLINITOS}

\section{PARALISIS DE LANDRY}

OBSERVACION' 1, -- M. A., dz 11 añ'os dz zfad, hija úniça, buen estado nutritivo. desarrolto neuro-psicológico normal. Estudiante de primer zño de Humbaidades.

Antecedentes hereditarios. - Padres aparentemente saros con Wass. (一). Sin antecedentes akohélicos ni tuberculosos. Ambos de constitución hipereno. fiva exagrada: pidre de temperamento paranoico, dado a la teosofía y al vegetatianismo. Siguiendo sus teorias, privó a su bija del tipo normal dz alimen1ación, rétringiéndole en forma considerable las albúminzs $y$ proporcionándele un exceso de hidiatos de carbono.

Antcicalentes personales. - A los 9 años presentó una cecarlatina de tipo benigno, que evolucionó sin dejar secuelas. A los 10 años sarampión. Amigdalitis a reperición.

Enfermedad actual. - Estanjo convalecientz de un cuadro gastrointesti11.1. lo: padrs sotan yu bace dos dias esta decaida $c$ inapetente. Al dia sij-i guicnte. la niña sale a cas, de una amiga; su madre, creyendo que se ha extraviado, sale a buscarla $y$ al traerla a casa, noca cierta torpeza al caminat, qua $s$ acompaña da debilidad de sus miembros inferiores, qque casi le imposibilizan c1 suhir la escala de su casa. Se mantiene estiz cuadro for al resto del día. hasta que la deambulación se haci imposible, acompañandose de doiores vagos a los miembros inferiores. En estas circunstancias es llevadia a la cama; sus familiarz: atribuyen el cusdro a un procaso reumático y se practicaron friegas.

A las 8 d. la mafiana ss examinada por uno de nosotros quien com. pruibi un chica pilida, con facies de suititinno, en postraćón absoluta. TemFratura axilai, 37,5". Llama la atección una paresia da los miembros superioro, con leatiou $y$ dificultad en los movimientos, fuerzas francamente disminuidas. Lon: refiejos tricipital y palmar muy débiles. En los miembros infelores hay franca parálisis. con hipotonía intensa, relajación muscular e hiper ustisia culán. Raflejos osteo-articulares abolidos. Babinsky regativo y no hay alisraciones de la sensibilidad. Junto a esto hay una franca hipctonia de los múseulos dorsales y de la nucu. Cofalea intensa.

Ex:minada 2 horas más tarde, nos encontramos con una niña en samo grado da postración, con enorme dificultad para los movimizntos parciales y tolales. Cetalea dun más intena y dificultad inicial para la respiración, que so revelaba for el esflerzo expiratorio. Cualquier movimiznto arrancaba quejum- 
arss di parte del entarmo y elinicamente no babia ninguna acritud díndole melodramática. qu pudiera hacet pensar en una impolencia de tipo funcionai. Prezentaba un discreto grado de sopor mental. Sus fuerzos estaban muy disminuidas en los miembros superiores, no aptetaba la mano $\dot{y}$ el acto de saludo era imposible; habia flacidea c hipotonia $y$ la arreflexia eta total. No babia alteracioacs de la sensibilidad. Babinsky negativo. A esa hora sa esboza $\gamma^{2}$ un escado de disfagia.

Sólo en la nectbe se pudo hacar la puación lumbar, debido a la tésistencia de la familia y cuando el cuadro habia avanzadn enormenente, Los examene: cnntinuajos do huta in hora indicaban el atmente de la impotencia liun. cional y sobre codo ta invasión ascendente dis mal. poss la disfinga: era tot.al in las idtionas horis de la carde y la dificultad respiratoria hacia times las asfixia. presentardo. al mismo tiampo. gran acumulación d: secreciones tráquio. faringias. Adamas. tenía afonia completa e imposibilidad para laser.

Se le practió una iransfusión s.nnguinea y se li colosó 90 migrs. de vi. tamiria Bl. Estimulantes periféricos y oxígeno. Aspiración d. las secteciones.

La enfermita sigue agravándose, durante la nothe, peimando el sindrome iespiratorio. caracterizado por la asfixia. La temperacura nunca pasó de $37.8^{\circ}$ Fil putso era blando $y$ rápido.

Fallece nuestra enferma a las 36 horis de iniciado el curdro.

El examen de fondo de ojo y campo vistal fui normal.

El restedo de: examen del liquido zéfalo-raquideo fué negarivo cabúmina. 0.20 gre por mit y 3 lucocitos por mm, 3 i.

Ccmentario. - En esta niña se hizo el diagnóstico de parálisis ascendente de Landry, tomando en consideración los siguientes datos:

1) Aramnesis: comienzo brusco. Falta de temperatura.

2) Cuadro clínico: parálisis rápidamente ascendente. fácida simétrica, con conservación de la sensibilidad. Compremiso de los músculos del tronco. Falta de trastornos esfinterianos.

3) Evolución: rápidamente fatal (36 horas).

4) Líquido céfalo-raquídeo: normal.

\section{PARALISIS DE LANDRY}

OBSERVACION 2. -- J. P., de 1 año 10 meses, con $15 \mathrm{~kg} r$. de peso. Sixo mastu;ino.

Antecedentes berditaitos. - Padres sanos, Wass. (-) en ambos. Dos Sijos: al otru as sano. Sin intecedentes tuberculosos.

Antexedcnte; personales. - Ha sida sano. 
Alímentación: pecho basca los 9 meses. Desde los 7 meses, una sopa: a los 10 meses, dos sopas. Desde esa edad leche condensada (alimentación controlada en el \$. O.J.

Enfecmedad acrosil. - Comienza hace 3 dias con vómitos post-prandiales, decaimiento, intranquilidad, irtitable. Al dia siguirnte sigue igual, llota por rualquiera cosa y anmque puede mantenerse en pir, se car; no tiene temperatura (T. R. 37.5\%). Se queja de dokorís abdominales. Consulta a.l $S . O$., donde Jẹ colocan un enernar por estar estitico, $y$ le indicaron aseptil. del que tomó sólo un gramo En la rarde de ese dia, no puede caminar. Es examinado por nno de nosotros.

Se compraba un niño con buen estado nutritivo, en posición pasiva. T. R., 37.8". Intranquilo, angestiado. Se encuentra parálisis flácidas de las cuatro extremidades. Arrefléxia tendinosa. Parálisis del diaftagma y cierto grado de inmovilidad de la rajilla costal. Intenso compromiso de los músedilos del tronco. Dificultad respiratoria. Disfagia.

Se pracricó una punción lumbar en la Posta Infantil, donde fué enviado.

Estavo hospitalizado ahi 24 horas y fué retirado por exigencia de la famitia,

Fallece al tereer día de iniciadas las parálisis.

El examen de líquido céfalo-raquideo fué negativo (albúmina, $0.20 \mathrm{gr}$. por mil, 5 leucocitos por mm.3).

Comentario. - En este chico hicimos el diagnóstico de parálisis de Landry, basándonos en:

1) Cuadro clínico: parálisis flácidas, simétricas, arreflexia tendinosa. Progresiva. Compromiso de los músculos del tronco. Falta de temperatura alta al iniciarse.

2) Evolución fatal. ciones.

3) Líquidic céfalo raquídeo: con mù leves altera-

\section{POLIOMIELITIS ANTERIOR AGUDA ASCENDENTE (HEINE-MEDIN)}

OBSERVACION 3. - Ficha 42/3477. Hospital Roberto del Rio.

T. P. de 6 meses de edad. con 5.990 grs. de priso y $62 \mathrm{cms}$. de talla,

Antecedentes bereditatios. - Padres sanos, Wass. (-). Siete hijos, nno murió de mevingitis y otto d. toberculosis. En la casa conviven 2 niños azon toberculosis.

Alimentación: pecho cada 3 . horas.

Enfermedades anteriores, -- Rinofaringitis, sarna, dermatitis seborreica.

Enfermedad actual: S: inicia bace 4 dias con decairniento, somnolencix. fiebre, transpiraciones profosics. Los dias dispaés la madre nota que el niño gtitaba al mudarlo. En la tarde no mueve las extremidades inferiores $y$ tiene 
convulsiones; el dia de a ingreso al hospital se le paralizan las extremidades supcriores; tieve, además, gran dificaltad para respirar. Le ha llamado la atención a la jojade que el niño, a pesar del estado de angustia que tenia, no llotuba desde áyer.

Examen física. - Lactante pálida, con facies tóxica, somnolienta. Cianosis ligera de los labios. T. R., 38.7\%"

Cabeza: cráneo, fontanela anterior tensa. Cata: ojos. pupilas mióticzs estrabismo convergente. Boca (-).

Cuello: discreta tigidez de la truco.

Tórax: parálisis de los músculos intercostales del lado dercho. Paráli is del diafragms, más acentuada al lado derecho. Corazón: tonos rápidos. Pu!. mones (-).

A.bdomen: parálisis dàe los músculos del lado detecho. Metorismo. Se palva un gran globo vestical. Higado y bazo (-). Se palpa el riñón izquierdo. Región ano-genital: nada de especial.

Extremidades: parálisis flácida con abolición de los reflejos en la extrsmidad superioc derecha e inferior izquierda, satwo el plantar quz está débil. Paresia en la extremidad inferior derecha con tonicidad más o menos cor. servada.

Erolución, - Como tratamiento sa le indicó vitamina B1 25 migrs. cada 6.horas. Oxígeno a petmanencia. Se trató de colocar el aparato de Drinket. pero è lactante era muY pequeño y no cumplia bien sa función. Practiçamos una raquicentrsis; el liquido obtenido fué claro.

El niño continuó agravándose. falleciendo a las 12 horss de ingreşs, debide al tolal compromiso de los músculos respiratorios.

En su estada fué necesario extraerle orina con cateterismo.

Comentario. - Este lactante fué enviado a la necronsia con el dịagnóstico de Heine Medin. Los fundamentos con que contábamos eran:

1) Anamnesis: sintomas prodrómicos manifiestos (traspiraciones, dolor al mudarlo, fiebre).

2) Cuadro clínico: presencia de parálisis flácidas, asimétricas, algunas zonas con compromiso parcial y otras con simple paresia.

3) Líquido céfalc raquídeo: con alteraciones frecuentes en la poliomielitis; albúmina, 0.40 gr.. por mil; Pandy. ++ : Ross Jones, +; Leucocitos, 212 por $\mathrm{mm}^{3}$; Linfocitos. $100 \%$.

En el examen anátomo-histo-patológico se comprobó ampliamente nuestra aseveración clínica. En los cortes de medula y bulbo, encontró el Dr. Guzmán, las lesiones carac. terísticas de esta enfermedad. Protocolo, 352/42. 


\section{POLIRRADICULONEURITIS AGUDA \\ (SINDROME DE GUILI.AIN'BARRE)}

OBSERVACION 4. - Hicha 1392/44. Hospital Robitto dil Rio.

N. P. de 1 año 8 meses di edad. ron 10,300 grs. di peso.

Antecedentes heredita jios, - Madre de 20 años, no se ha hicho ración d: Kahn; hace 2 ajos iuvo un proceso tuberculoso del palmón détecho. Pa. dre de 25 años, aparentemente sano. tampoco tienz teacciones serológicas. Dos hijos vivos, al otro es mesor y actoulmente sano.

Antecedentes personales. - Nació en parto normal, di término. en la casa (Vallenar).

Alimentación: Picho + mises. Desputs Milko en buena proporción. Sopas y purés a los 8 meses. Leguminosas a los 9 meses. Carnes $y$ huevos a los 13 mess; y a esta edad lambien recibia $200 \mathrm{grs}$, de leche de vaca. 3 vecas en el día. Jugo de ftutus desde los 4 meses.

Desarrollo: afitmó la cabeza a los 4-meses. Se tria deste el sazundo. A los 8 meses se sentaba $y$ a los 11 andavo; a esta edad pronunciaba paladoras.

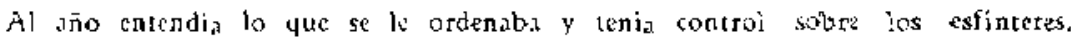
Dënición a los 7 meses.

Enfermedades anteriores. - Dispepsia a veces. Tiez en la cata. Rabietas. En seneral es may nerviosa.

Enfermedad actual. - Más o menos a firies de maszo del presinte año, soservaron en la chica un cambio de caracler notable: jasaba de muy mal penio z irritable. traspiraba mucho en las noches. Veinle dias antes de inisiars a caldro que destribiremes más adchonk, la niña tenja menos firmeza en las piernas, se caia con frecuencia: su abuela decia yue Normita tenía las "piernas de lana": tenia catambres. decia la madre. En lits tardes y noches afusaba dolores. por to que le pracljaban frizgas. E1 dia 11 de abril. los do. lores se hacin muy intensos en los brazos y piemas, se agrega insomnio, traspiración profusa. con congestión intensa de la cara (coloradita). En estas con. dicjones pasó hạsta el dia 14 . en que amanece con los miembrọs inferiotes compleraminte inmóviles. Al dia siguiente fueron las manos $y$ al subeiguiente jos antebrzzos y brazos. El dia 16 presenta sonquera y gran díficultad para respirar. E1 19 se agrava aún mis. se pone bianotica, con marcada asfixia. respiración estertorosa, con gran acumulación de secreciones. Los padres, dasespe. rados. toman un avión $y$ la trastadan a numbtro Servicio.

Examen de ingreso. - Lactinte con buen estado nutritivo, en posición pasiva. Piel húmeda, sudoross. congestionada. Elasticidad disminuida. Cianosis do la cara y uñas. Respiración esierterosa. dificultosa, artitmica, exclusivamenic costal. Pulso muy débil. incontuble. Temperatura reśas. 39.5?.

Cabeza: cráneo: nada de especial. Casa: ojos. extrabismo convirgente: pupilas, iguales. reacionan bion a la luz: boca. lengua saburtal: faringe. rojs. con grar acumulación mucosa réronasal: dentadurá. co hica estado. 
Curtlo: dolor al flectar lu sabeza. Parálisis de los mústulos anteriores dit ciello.

Tórax: fa incursión respiratoria sc rextiza cáj sxclusivamente a base de los intercostales. Abombamiento de las bises colo absoluta inmovilidad diat fragmárica. Corazén: tonos rápidos apagaclos. Pulmones roncus de virtices a bas: en ambos campos.

Abdomen: higato a 3 cms. del riborde. Bazo y riñones no se palpan.

Región zno-genital: ruiva enrnjecida. con secruien purulenta.

Lxtramidades: parálisis flácda cotal de las superioris e inferiorse Hiper-

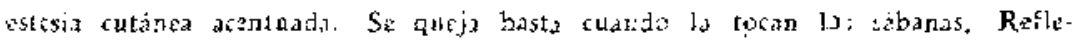
jes tendinosos obolidos. Movimientos pasives dolorosos.

Psiquis: sensorio despejado cón traduiz ya estado di angustia mareado.

No bay compromiso de los musculos abdominales ni interostales.

Evolución y examenes. - A su ingrist s: particó una putación lasubar, satiendo liquido clato gola a gota- Como liatamiento sa le indicó: posirión de Trindelenburg. Inmovilidad da los niemboj en buina posición. con kacos de arena, Calsntamiento de zilcis. Piza istada. Aspiración de las sccreciones traqueo-faringeas. Inmovilidad absoluta. pues. al menor movimuto tenia crisis de asfixia. Alimentación con sonda esofigica. pues no pofil deglutir, Como tratamiento medicamentoso se la indicó coramia cada 4 hora; $y$ vitamina $B, 10 \mathrm{~m}$ lgrs. 2 veces.

Fo las condiciones deseritas pasó 3 dias: en ocasiones. la aspiración de las secrecionts tuvimos que bacerla piriondmente y las crisis de asfixia cran muy frecuentes, de abi que toviera gue tener ura enfimera plitmanentemente a su lado. Al tercer dia cayó estoontanesmont: la limparatura y sa podia apreciar un signo de buen pronóstico, movía mu ligaraments los ortejos. $l$ a parálisis edpezaba a regresar. Al sexto dia funcionaba el diafragima. Estátamos ya francamente optimistas. cuando la chica presintu un, al7a de temperatura a 40 grados, polipnéa y dicainiecto inteuso: sólo se encontró una laringe roja $y$ estertores catarrales diseminados en appos campros pulmpnaxes. A Prsar del temor que tríanios a una bronconeumonia por aspiación o eciásica. no indicamos quimioterapia. nos limitamos a esperar. alamentando sólo las dosis de cotamina. Afortunadamente. el cuadto cedió en tros dias. Fur uara zripe, que la toletó bien.

Posteriomente continuaron regresindo las ror :s paralizadas y al mes de sstada, movia los hombros. flaclaba las rodillas y los cados. Estos movimienros fueren hatiéndos cada dia con más fuerza y en mís extensión. A los 45 dias de enfermedad. podia saludar, sentarse y se aimonaba sola.

Dos puntos debemos anotar en la evalución de erta cofermedad:

1) La patálisis regrasó en sintido invirso it que apareció. es derir. primero ies segmentos proximales, juego los distales.

2) Vo se produjo atrofia muscular an ringún segmento.

Los dolors pasaron por complato 210010 días de iniciado el trata miesto. 
En el líquido céfalo-ralquideo se encontró lo siguiente:

Incoloro.

Aspecto: transparente, con péqueños gtumos fibrinosos.

Albúmina: 1 gr, por mill.

Pandy: +++ .

Ross-Jones: ++ .

Cloruros: 6.80 grs. por mil.

Leurocitos: 3 por mm.s.

Es dair. había una notable disociación albúmino-citológica.

Otros exámenes que se practicaton, mostraton:

Orita, Kabn y secreción vaginal (-). Mantoux al 1 x 1.000 (-).

Hemograma: leucocitosis de 25,600 , con gran desviación a la izquierda; segm zntados, 39: baciliformes, 22; juveniles, 1; basófilos, 1; jinfocitos, 28; inonocitos. '8. Hemaries, 5.900,000.

Velocidad de sedimentación, $7 \mathrm{mms}$. en una hora.

A los 28 días se repitió 1 a raquicentesis y el examen del lisuido dice: Incolaro.

Aspecto transparente.

Pandy: ++ .

Cloruros: $7.10 \mathrm{grs}$. por mil.

Abbúmira: 0.35 gr. por mil.

Leacocitos: 1 por $\mathrm{mm}^{*}$.

Comentario, - En resumen, se trata de un lactante de un año y ocho meses, que ingresa al hospital con un sindrorme de parálisis ascendente, precedido de un largo períado dolotoso y de hipotonía. Cuadro que evolucionó a la curación total después de dos meses. En los primeros días, el estado de enorme gravedad que presentaba y sobre todo el inminente peligro.de asfixia, noos hacía ser pesimistas. Las parálisis regresarcn en orden inverso al que aparecieron y no se produjeron atrofias musculares.

En este lactante hicimos el diagnóstico de Sindrome de Guillaín Barré, considerandò:

1) Anamnesis: antecedentes de dolores en las extremidades desde varios días atrás. Hipotonía.

2) Cuadro clínico: parálisis ascendente, simétrica, con compromiso de los músculos de las cuatro extremidades en totalidad. Falta de compromiso en los abdominales. Arreflexia tendinosa, con conservación de la sensibilidad cutánea, que estaba francamente aumentada.

3) Disociación albúmino-citológica en el líquido céfalo-raquídeo, comprobada en dos ocasiones. 
4) Evolución favorable de la enfermedad.

Ccmo comentario final diremos que éste es el primer caso de polirradiculoneuritis aguda, en un lactante, que se da a conocer en nuestro país y que presenta los síntomas cardinales que se atribuyen a esta enfermedad.

Gareisso y Sagretas, en 1938, publicarcn el primer caso de Guillain-Barré. en Argentina; tenía etiología bien precisa (difteria) y posteriormente agtegaron otra abservación de etiolczía tuberculosa (1942). Casaubon y Puglisi, en el mismo año. comunicaron también un caso de etiología dudosa, probablemente un procesa ótico podia actuar en ét, cuando menos como factor desencadenante.

Feluffe. en Uruguay (1939), describe un cuadro semejante, pero de etiología tífica. En el mismo país, Portillo y Malosetti (1942) dieron a conocer un caso de GuillainBarré en un niño de 11 años de edad, de etiología desconocida, en el que llamaba la atención la gran cantidad de a!búmina en el líquido espinal ( 6 grs.).

\section{REXSUMEN}

Los autores presentan cuatro casos de parálisis ascendente aguda. Dos casos de Parálisis de Landry. 1 de poliomiclitic ascendente y 1 caso de polirradiculoneuritis aguda o sindrome de Guillain-Barré.

Hacen un estudio de cada uno de ellos, insistiendo principalmente en el diagnóstico diferencial, recalcando la importancia que tiene la anamnesis, cuadro clínico, principalmente en lo que se refiere a simetría y distribución de las parálisis. y el examen del líquido céfalo-raquídeo; en las poliomielitis con su característica reacción: albumincsis, con pleacitosis (lintocitosis) : en el sindrome de Guillaint-Barré observar to que es patognomónico de esta afección. discciación albúmino-citólógica, Pot último, en la Parálisis de Landry, son catcgóricos en afirmar la ausencia de toda reacción inflamatoria o albuminosa.

También hacen nctar la diferente evolución en estos cuadros en apariencia semejante: en el Landry, fatal en su gran mayorja, y en el sindrome de Guillain-Barré afirman ¿a buen pronóstico, con tendencia a regresar en su totalidad, a pesar de lo alarmante de la enfermedad.

Afizman no haber encontrado en la literatura nacional un casa de Guillain-Barré descrito en un lactante. 
Finalmente hacen un estudio de la parálisis ascendente aguda, con especial referencia a la parálisis de Landry, con el objèto de fijar normas de diagnóstico, pronóstico y tratamiento en las parálisis ascendente. Cogen ellos la experiencia de autores extranjercs, con especial referencia a la Escusla Americana.

\section{Bibliografía.}

1.-BODIAN D.. HOW'E A. - Proc Soc. Exp r. Bioj. y Med. 41: 540: 1939

2.-BOX. C. R. - Iancer: 1: 22: 1921.

?--BRENEMANN. - Practice of Pediatric. Tomo IV-17: 1942.

4.-CASAUBON A., PUGLISI A. - Arch. Arger. Ped. 18: 99: 1942.

5.-FORD. F. R. - Bull. J. Hopkins Hosp. 43: 140: $19 \geq 8$.

G.-GUJt LAIN. BARRE. STROAHL. - Bull. y Mém. de la Soc. de Múd. de 'Hop. de Paris. J916..

7 -GREENFIELD. J. G. and BUZZARD. - Pahology of th: Nervons Systetn, 209; 192?.

8.--GRUNEWALD. E. A. - J. Psychal. und Neurol. 39; 55: 1923.

9.-HOLI y HOWLAND. - Tratado de Pediatria. Tomo 1I-1301.

10.-KNUTTI. R. E. - J. A. M. A. 93; $754: 1929$.

11.-LANDRY. J. B. -.. Gaz. Hébd. de Med. Paris. 1859

12-1 ANDSTEINER y LEVADITI - Ann. de LInst. Pasteur. 23; 83.3: 1910.

13.-MAC CARROI.. - J. A. M. A. 120:517: 1942.

14.-WEI.SON. -- Encicl. Tomo IV -547 I.

15.-PELUTFO E. - Arick, de Ped. Utuguay. 11: 3: 1940.

ik.-- PORTLlo J. M. y MALOSSETTI. - Arch. de Ped. Uruguzy, 12: $163: 1942$.

17--REMLINGER, - Ann. Inst. Pasteur. 47; 71: 1928.

18.-ROLLESTON. - Arth. Pediat. 30: $335: 1013$.

J 9.-SABIA A. - J. A. of Ped. 21; 353; 1942.

2C.--SAPAS. E. A. - J of Psichiat and Neut. 42; 151: 1923.

21.-SMIR.NOW. L. If - Arch. f. Psichiat und Ners. 78; 585: 1926.

22.-UNGER L. - Trarado da Enfermedades de los Níños. Pig- $5+3$.

23.--THORNER. ALPERS. YASKIN' - Arch. Neur artal Psychiai. 4?: $17 \cdot 42: 1940$. 